\title{
Regeneration Savings in Coherent Optical Networks with a New Load-dependent Reach Maximization
}

\author{
A. Bononi(1), P. Serena ${ }^{(1)}$, A. Morea ${ }^{(2)}$
}

(1) Università di Parma, Dip. Ing. dell'Informazione, via G. Usberti 181/A, Parma (Italy), $\bowtie$ bononi@tlc.unipr.it (2) Alcatel-Lucent Bell Labs France, Nozay (France).

\begin{abstract}
We propose a new load-dependent reach maximization procedure in dispersionuncompensated optical networks with coherent detection, and estimate the electro-optic regenerations savings with respect to the standard full-load reach approach.

\section{Introduction}

We consider here the physical layer design of flexible optical networks, where dispersionuncompensated (DU) wavelength division multi-

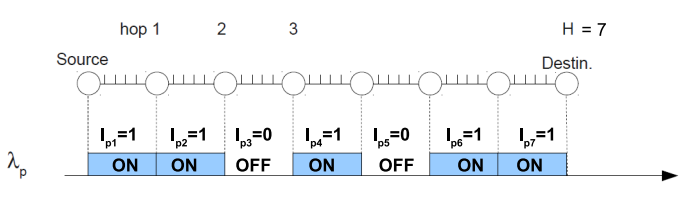
plexed (WDM) dual-polarization (DP) optical digital signals are transmitted and coherently detected. From the source, the destination may be transparently reached via a single lightpath without electro-optic regeneration (EOR), or through a concatenation of lightpaths on possibly different wavelengths, with EOR from one lightpath to the next one. To minimize the number of costly EORs, the quality-of-transmission aware routing and wavelength assignment (RWA) algorithm first tries to set-up a circuit along a single lightpath. Connection may be unfeasible for two reasons: i) unavailability of the same wavelength across successive fibers along the lightpath, leading to wavelength blocking (WB); ii) the received signal to noise ratio (SNR) for the considered modulation format is below a required minimum $S_{0}$, leading to SNR blocking (SB).

We concentrate here on SB due to accumulation of linear and nonlinear optical impairments. The standard approach is to set-up only lightpaths whose physical length is below the full-load $(F L)$ reach ${ }^{1}$, i.e., the maximum length guaranteeing a received SNR above $S_{0}$ when all $W$ wavelengths on all fibers are occupied. The FL reach is used regardless of the actual wavelength load $u$, i.e., the fraction of network wavelengths actually utilized by set-up lightpaths. Using the FL reach is clearly conservative, since wavelengths saturation at the network core prevents the average network load $u$ to reach unity. In this paper, we propose a new power selection strategy that maximizes the reach at the actual load $u$, and quantify the potential EOR savings with respect to using the $F L$ reach and the power selection strategy in ${ }^{1}$.

\section{Nonlinear transmission with ON/OFF traffic}

Focus on a reference lightpath from source to destination, composed of $H$ hops across access nodes, where the $k$-th hop is a concatenation of
Fig. 1: ON-OFF lightpath process on $p$-th wavelength.

$\mathcal{S}_{k}$ amplified spans followed by the crossing of the $k$-th intermediate node, for $k=1, \ldots, H$. A span consists of a transmission fiber followed by a lumped optical amplifier. A node is composed of a wavelength demultiplexer, add/drop block and output multiplexer. The lightpath is composed of $N_{s}=\sum_{k=1}^{H} \mathcal{S}_{k}$ spans. Interfering traffic is modeled by assuming that each of the $W-1$ remaining wavelengths of the $k$-th hop independently carries a lightpath (hence power) with known probability $u_{k}, k=1, \ldots, H$. Within a first-order regular perturbation analysis, the received SNR over the bandwidth of the DP signal of interest after propagation across the reference lightpath is ${ }^{2}$ :

$$
S N R\left(P, N_{s}, \mathbf{u}\right)=\frac{P}{N_{A}+a_{N L}\left(N_{s}, \mathbf{u}\right) P^{3}}
$$

where $P$ is the DP reference lightpath power at the input of each transmission fiber section; $N_{A}$ is the amplified spontaneous emission power which scales linearly with $N_{s} ; a_{N L}=a_{N L}^{S C I}+$ $a_{N L}^{X C I}$ is the nonlinear interference (NLI) coefficient ${ }^{2}$ contributed by single- and cross-channel interference $(\mathrm{SCl}, \mathrm{XCl})$. While $a_{N L}^{S C I}$ is deterministic, we can prove ${ }^{3}$ that in DU links $a_{N L}^{X C I} \cong$ $\sum_{p \neq 0} \mathcal{C}_{p} \sum_{k=1}^{H} \mathcal{S}_{k} I_{p k}$, where $\mathcal{C}_{p}$ is a link- and pump-dependent coefficient at wavelength $\lambda_{p}$, and the indicator random variable (RV) $I_{p k}$ equals 1 (with probability $u_{k}$ ) if a lightpath is $\mathrm{ON}$ at $\lambda_{p}$ at hop $k$, and 0 otherwise, as sketched in Fig. 1. Hence the $a_{N L}$ coefficient and in turn the received SNR are RVs, whose statistics depend on the load vector $\mathbf{u}=\left[u_{1}, \ldots, u_{H}\right]$. The digital signal has a forward error-correction code whose SNR threshold (plus margin) for the signal modu- 


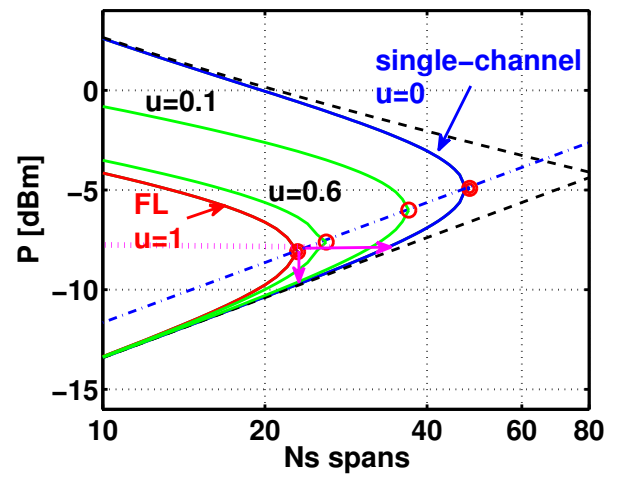

Fig. 2: Contours of SNR-blocking probability at level $\mathcal{P}_{S B}=10^{-3}$ versus power $P$ and number of spans $N_{s}$ at load values $u=[0,0.1,0.6,1]$. All pairs $\left(P, N_{s}\right)$ inside each contour yield $\operatorname{Pr}\left\{S N R\left(P, N_{s}, \mathbf{u}\right)<S_{0}\right\} \leq \mathcal{P}_{S B}$, with SNR over signal bandwidth $S_{0}=9.8 \mathrm{~dB}$ (DP-QPSK at BER $=10^{-3}$ ).

lation format is $S_{0}$. We declare an SB event when $S N R<S_{0}$.

The design of point-to-point DU transmission systems for DP WDM coherent systems is based on the received SNR contours versus number of spans $N_{s}$ and transmitted power $P$ (assumed here the same for all signals). In a networking scenario, however, the SNR is a RV. We propose here to base the design of DU networks on contours of the SB probability $P_{S B} \triangleq$ $\operatorname{Pr}\left\{S N R\left(P, N_{s}, \mathbf{u}\right)<S_{0}\right\}$ at fixed load $\mathbf{u}$ versus both power per channel $P$ and number of spans $N_{s}$. The proposed load-dependent RWA, which needs only knowledge of the load vector $\mathbf{u}$, declares that a new lightpath of length $N_{s}$ has sufficient SNR at destination if $P_{S B}$ is less than or equal to a target level $\mathcal{P}_{S B}$ for the selected modulation format. All details of the SB probability derivation from the statistics of the modulationformat-indepenent $a_{N L}$ from the Gaussian Noise (GN) model ${ }^{2}$ are presented in ${ }^{3}$.

\section{Results}

From the $P_{S B}$ contours at the target level we visualize both the maximum number of spans that can be bridged without EOR (i.e., the maximum load-dependent reach) and the associated optimal power. In the numerical calculations we assumed the spans are identical, the load $u_{k}$ and the spans per hop $\mathcal{S}_{k}$ are uniform at all hops $k=$ $1, \ldots, H$, with $\mathcal{S}=2$ spans per hop, and all signals have the same format (i.e., power and bandwidth), although the theory is developed for nonuniform $u_{k}, \mathcal{S}_{k}{ }^{3}$ and can be extended to mixed modulation formats. Fig. 2 shows the SB probability contours at a target electrical SNR $S_{0}=$
$9.8 \mathrm{~dB}$ (over the matched-filter bandwidth, yielding a $10^{-3}$ bit error rate (BER) for DP quadrature phase shift keying (DP-QPSK)), for $W=81$ wavelengths and $R=10$ Gbaud signals transmitted with spacing $\Delta f=12.5 \mathrm{GHz}$ (bandwidth efficiency $\eta=\frac{R}{\Delta f}=0.8$ ) over $N_{s} 100 \mathrm{~km}$ DU spans (dispersion $D=2 \mathrm{ps} / \mathrm{nm} / \mathrm{km}$, attenuation $\alpha=0.2 \mathrm{~dB} / \mathrm{km}$, nonlinear coefficient $\gamma=1.3 \mathrm{~W}^{-1} \mathrm{~km}^{-1}$ ) and amplifiers noise figure $F=4 \mathrm{~dB}$. The points of maximum reach at the optimal power are marked by red circles in the figure. We indicate their coordinates as $\left[N_{0}(u), P_{0}(u)\right]$. At $u=1$ and $u=0$ the SB contours at all $\mathcal{P}_{S B}$ levels coincide. For all $\left(P, N_{s}\right)$ pairs inside the region delimited by the red contour (at $u=1$ ) or blue contour (at $u=0)$ the SB probability is zero, while outside it is 1 . Instead, at any intermediate load $0<u<1$ the contours vary with the value of $\mathcal{P}_{S B}$, and all $\left(P, N_{s}\right)$ pairs inside each contour yield $\operatorname{Pr}\left\{S N R\left(P, N_{s}, \mathbf{u}\right)<S_{0}\right\} \leq \mathcal{P}_{S B}$. For instance, at loads $u=0.6$ and $u=0.1$ the green lines show the contours at level $\mathcal{P}_{S B}=10^{-3}$. The locus of maximum reach points, as $u$ varies, can be shown to lay on the dashed-dotted straight line shown in Fig. 2 parallel to the (lower) linear asymptote and shifted by $10 \log (3 / 2) \cong 1.76 \mathrm{~dB}$ above that.

In Fig. 2 the linear asymptote and hence the dashed-dotted line have slope $1 \mathrm{~dB} /$ decade, hence the magenta arrows in the figure indicate $1.76 \mathrm{~dB}$ on each axis direction. This has a fundamental consequence, first noted in ${ }^{1}$. If we fix $P$ to the full load optimal value $P_{0}(1)$ (magenta dotted line) then the ratio between the FL reach $N_{0}(1)$ and the reach at any other load $u<1$ is always smaller than 2/3. Thus, if in the RWA algorithm we use the FL reach $N_{0}(1)$, at most we underestimate the true reach by a factor $1 / 3$, i.e., by $33 \%{ }^{1}$. This was the rationale for proposing the $\mathrm{FL}$ RWA design that uses the distance-independent power $P_{0}(1)$ in ${ }^{1}$. However, suppose for instance the actual load is only $u=0.1$. If we use the true maximum reach power $P \equiv P_{0}(u)=-6 \mathrm{dBm}$ (see contour at $u=0.1$ ) we find that the maximum reach is $N_{0}(u)=37$ spans, which compared with the FL reach $N_{0}(1)=23$ spans gives a reach under-estimation by the FL RWA with respect to the proposed load-dependent RWA by: $\mathcal{U} \triangleq \frac{N_{0}(u)-N_{0}(1)}{N_{0}(u)} 100=37.8 \%$, which is above $33 \%$. This means that if we know the average wavelength load $u$ and then select the maximumreach power $P_{0}(u)$, the under-estimation with respect to the actual reach $N_{0}(u)$ when adopting the FL RWA can be larger than 33\%. The optimal power $P_{0}(u)$ and the corresponding reach $N_{0}(u)$ 


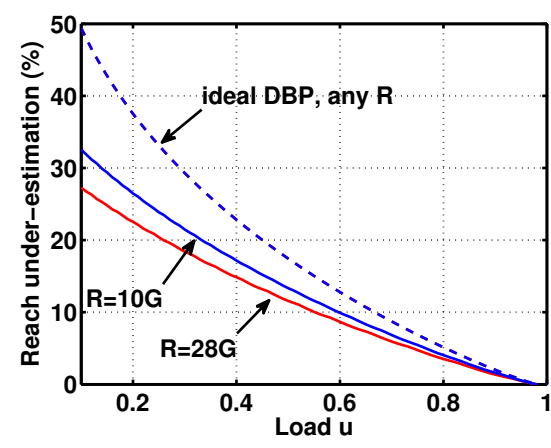

Fig. 3: Reach under-estimation $\mathcal{U}$ of FL $\mathrm{RWA}^{1}$ with respect to proposed load-dependent RWA versus load $u$ in a DU SMF link $(D=17 \mathrm{ps} / \mathrm{nm} / \mathrm{km})$ with $W=81$ WDM DP-QPSK $\left(S_{0}=9.8 \mathrm{~dB}\right)$, at SNR blocking probability $\mathcal{P}_{S B}=10^{-3}$, with $100 \mathrm{~km} / \mathrm{span}, \mathcal{S}=2$ span/hop, $\eta=\frac{R}{\Delta f}=0.8, F=4 \mathrm{~dB}$.

can be analytically derived at any load $u^{3}$. The reach under-estimation $\mathcal{U}$ turns out to be a decreasing function of dispersion $D$, symbol rate $R$, and load $u$. Fig. 3 shows $\mathcal{U}$ versus load $u$ for DPQPSK at both $R=10 \mathrm{Gbaud}$ and $R=28 \mathrm{Gbaud}$ on standard single-mode fiber (SMF, $D=17$ $\mathrm{ps} / \mathrm{nm} / \mathrm{km}$ ), both without and with ideal digitalbackpropagation (DBP). With ideal DBP only XCl is left $\left(a_{N L}=a_{N L}^{X C I}\right)$ and the reach $N_{0}$ is independent of channel symbol rate and just depends on bandwidth efficiency $\eta$. $\mathcal{U}$ is below $\cong 20 \%$ in all practical cases on SMF links at loads above 0.4.

We next need to quantify the savings in EOR when using the load-dependent RWA. A quick estimation is obtained as follows. We get the distribution of the lightpath length $N_{s}$ (spans) in the network from simulations when SNR blocking is neglected. Each circuit is set up on a single lightpath until the first WB, when the measured load is $u$. Let the topology-dependent simulated normalized histogram of lightpath lengths $N_{s}$ be $\mathcal{P}\left(N_{s}, u\right)$. We can thus estimate the expected number of required EOR when the reach is $N_{0}$ as $E\left[\mathrm{EOR} \mid N_{0}\right]=\sum_{N_{s}=1}^{N_{\max }} \mathcal{P}\left(N_{s}, u\right)\left(\left\lceil\frac{N_{s}}{N_{0}}\right\rceil-1\right)$, where $N_{\text {max }}$ is the maximal $N_{s}$ in the network, and $\lceil x\rceil$ is the ceiling function. The percent savings $\mathcal{R}(u)$ in EOR operations using our load-dependent RWA with respect to the full-load RWA is:

$$
\mathcal{R}(u)=\frac{E\left[\operatorname{EOR} \mid N_{0}(1)\right]-E\left[\operatorname{EOR}_{\left.\mid N_{0}(u)\right]}\right.}{E\left[\operatorname{EOR} \mid N_{0}(1)\right]} \cdot 100 .
$$

Note that whenever $N_{0}(1)<N_{\max }<N_{0}(u)$ the savings are $100 \%$, since no regenerations are required with the load-dependent RWA. Fig. 4 shows EOR savings $\mathcal{R}$ (red) and under-estimation $\mathcal{U}$ (blue) versus target SNR $S_{0}$ (i.e., modulation format) at the first WB load $u=0.46$ in a 46-node US network ${ }^{4}$ in uniform traffic, at $R=28 \mathrm{Gbaud}$ on SMF fiber, both with (solid) and without

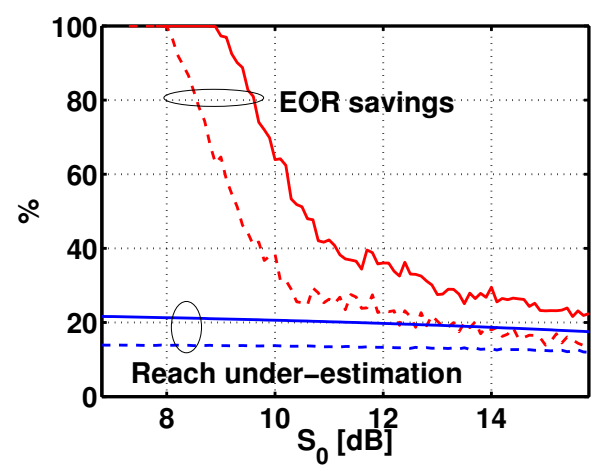

Fig. 4: EOR savings $\mathcal{R}$ (red) and under-estimation $\mathcal{U}$ (blue) versus target SNR $S_{0}$ (i.e., modulation format) at the first-WB in the US network ${ }^{4}$, at $R=28$ Gbaud $^{2}$ on SMF fiber. Other data as in Fig. 2. Solid: ideal DBP. Dashed: no DBP. $\mathcal{R}$ and $\mathcal{U}$ averaged over 100 simulations up to first WB; average load $u=0.46$.

(dashed) ideal DBP. We note that at the smallest $S_{0}$ of $6.8 \mathrm{~dB}$ (corresponding to DP binary phase shift keying at $B E R=10^{-3}$ ) no regenerations are needed in the US network even using $N_{0}(1)$, hence $\mathcal{R}$ is undefined. As we increase $S_{0}$ we go to a situation where $N_{0}(1)<N_{\max }<N_{0}(u)$, yielding $100 \%$ savings. As the modulation levels increase the required $S_{0}$ increases (e.g., $S_{0}=$ $15.8 \mathrm{~dB}$ for DP 16 quadrature-amplitude modulation), hence $N_{0}$ decreases, and the \%EOR savings $\mathcal{R}$ decrease towards the values of underestimation $\mathcal{U}$. Thus under-estimation is also a reasonable indicator of \%EOR savings only for higher-order modulation formats.

\section{Conclusions}

We have analyzed the potential EOR savings when using a load-dependent reach in place of the standard full-load reach ${ }^{1}$. For a 46 -node US network in uniform traffic over SMF links we find a reduction from $40 \%$ (no DBP) to $60 \%$ (ideal DBP) EOR operations at the load of first wavelength blocking $(u=0.46)$ for a DP-QPSK format. Higher-order modulations show smaller savings.

\section{References}

1 P. Poggiolini et al., "The LOGON Strategy for LowComplexity Control Plane Implementation in NewGeneration Flexible Networks" Proc. OFC'13, paper OW1H.3.

2 P. Poggiolini, "The GN Model of Non-Linear Propagation in Uncompensated Coherent Optical Systems," J. Lightw. Technol. 30, 3857 (2012).

3 A. Bononi et al., "Load-aware Transparent Reach Maximization in Flexible Optical Networks," Proc. NOC'14, Milan, (2014).

4 A. Morea et al. "QoT function and $A^{*}$ routing: an optimized combination for connection search in translucent networks,", J. Opt. Netw. 7, 42 (2008). 